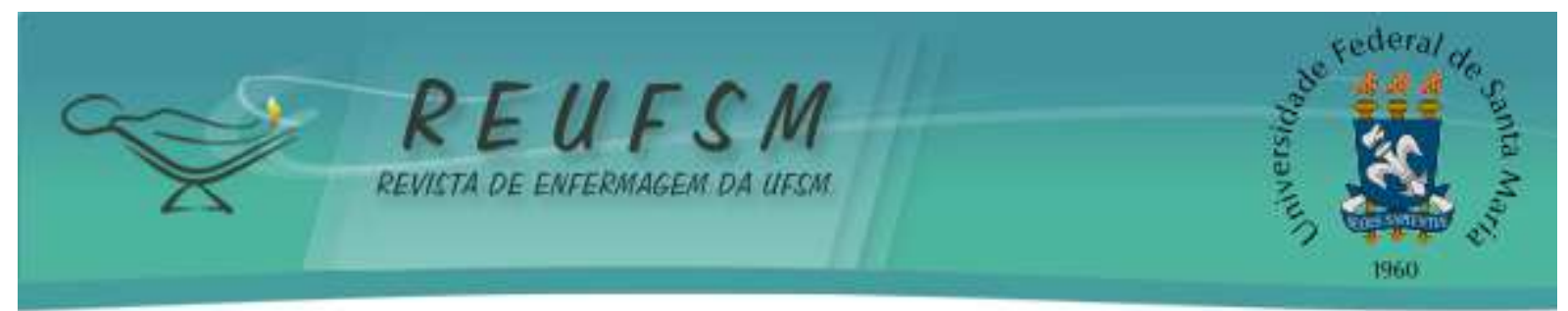

\title{
OFICINAS DE ESTIMULAÇÃO COGNITIVA PARA IDOSOS COM QUEIXA SUBJETIVA DE MEMÓRIA E HUMOR
}

\section{COGNITIVE STIMULATION WORKSHOPS FOR ELDERLY PEOPLE WITH SUBJECTIVE MEMORY AND HUMOR COMPLAINTS}

\section{TALLERES DE ESTIMULACIÓN COGNITIVA PARA ANCIANOS CON QUEJAS SUBJETIVA DE MEMORIA Y HUMOR}

\author{
Rosimere Ferreira Santana ${ }^{1}$ \\ Shardelle Araújo Alexandrino ${ }^{2}$ \\ Thais da Silva Soares ${ }^{3}$ \\ George Luiz Alves Santos ${ }^{4}$ \\ Elisa Monteiro Magalhães Bamberg ${ }^{5}$ \\ Thais de Medeiros Oliveira ${ }^{6}$
}

\section{Doi: $10.5902 / 2179769231200$}

RESUMO: Objetivo: analisar um programa de oficinas de estimulação cognitiva para idosos com queixas subjetivas de memória e humor e associá-lo ao desempenho da capacidade funcional. Método: estudo quase-experimental, quantitativo, realizado em um programa para idosos. Utilizaram-se como testes de seguimento: Mini Exame de Estado Mental (MEEM), Escala de Depressão Geriátrica (EDG), Escala de Atividades Instrumentais de Vida Diária (Lawton), Teste de Fluência Verbal (EVP) e Teste do Relógio. Análise dos dados descritiva e inferencial com a utilização do software R. Resultados: após as oficinas de estimulação cognitiva notou-se discreto aumento nos escores do MEEM e diminuição na EDG. Já nos escores dos testes Lawton, Teste do Relógio e EVP houveram manutenção nos valores, o que pode ser um indicativo de preservação da capacidade funcional. Conclusão: recomenda-se investir em programas para a terceira idade com protocolos de intervenção sistematizadas, que garantam atendimento especifico e seguimento de sua performance.

Descritores: Terapia cognitiva; Saúde do idoso; Transtornos do humor; Enfermagem geriátrica; Cuidados de enfermagem

ABSTRACT: Aim: to analyze a program of cognitive stimulation workshops for elderly people with subjective memory and humor complaints and to associate it with the functional performance of functional capacity. Methods: this is a almost-experimental, quantitative study conducted in a university extension program for elderly people. For

\footnotetext{
${ }^{1}$ Enfermeira, Pós-doutorado, Bolsista de Produtividade PQ-2, Professora Associada, Universidade Federal Fluminense. Niterói, Rio de Janeiro, Brasil. rosifesa@gmail.com

${ }^{2}$ Enfermeira, Ex-bolsista de Extensão do Projeto Memória Cognitiva, Universidade Federal Fluminense. Niterói, Rio de Janeiro, Brasil. shardelle@gmail.com

${ }^{3}$ Enfermeira, Mestranda em Enfermagem, Programa Acadêmico em Ciências do Cuidado em Saúde, Universidade Federal Fluminense. Niterói, Rio de Janeiro, Brasil. thaissoares@id.uff.br

${ }^{4}$ Enfermeiro, Mestre em Enfermagem, Programa Acadêmico em Ciências do Cuidado em Saúde, Universidade Federal Fluminense. Niterói, Rio de Janeiro, Brasil. georgealves.enf@gmail.com

${ }^{5}$ Enfermeira, Mestranda em Enfermagem, Programa de Mestrado Profissional em Enfermagem Assistencial, Universidade Federal Fluminense. Niterói, Rio de Janeiro, Brasil. elisamonteirom@gmail.com

${ }^{6}$ Enfermeira, Ex-bolsista de Extensão do Projeto Memória Cognitiva, Especialista em Enfermagem Gerontológica, Universidade Federal Fluminense. Niterói, Rio de Janeiro, Brasil. thaismeoli@gmail.com
} 


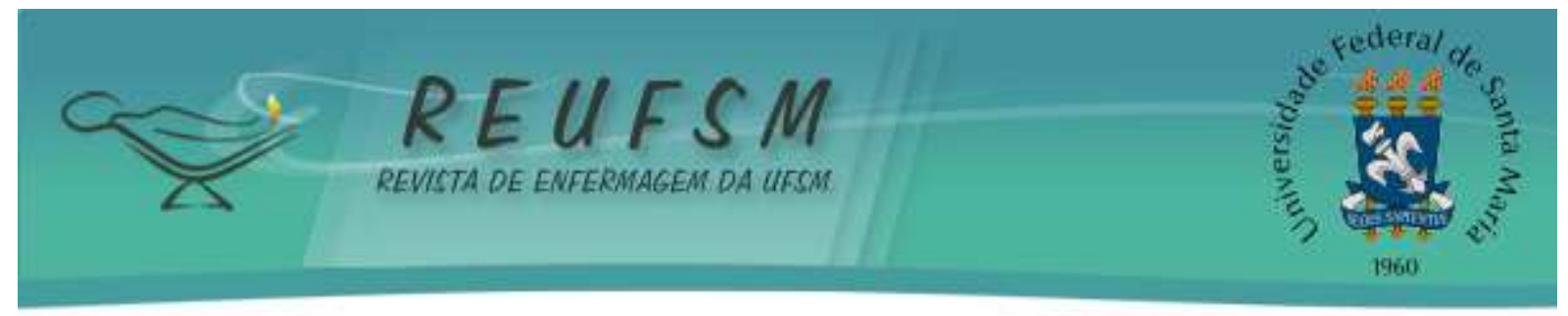

data collection, the following tests were used: Mini Mental State Examination (MMSE), Geriatric Depression Scale (EDG), Daily Life Instrumental Activities Scale (Lawton), Verbal Fluency Test (EVP) and Clock Test. Descriptive and inferential data analysis using $R$. software. Results: after the cognitive stimulation workshops, we obtained a discreet increase in the MMSE scores and a decrease in EDG. As for the Lawton Clock and EVP test scores, there were maintenance on values, which may be indicative of maintaining functional capacity. Conclusion: we recommend programs of cognitive stimulation with systematized intervention protocols, with specific attention to the demands of the elderly people and follow-up of the functional capacity.

Descriptors: Cognitive Therapy; Health of the Elderly; Mood Disorders; Geriatric Nursing; Nursing Care

RESUMEN Objetivo: analizar un programa de talleres de estimulación cognitiva para ancianos con queja subjetiva de memoria y humor asociado al desempeño de capacidad funcional. Método: estudio cuasi experimental, cuantitativo, realizado en un programa para ancianos. Utilizaron instrumentos de seguimiento: Mini examen del estado mental (MEEM), Escala de depresión geriátrica (EDG), escala de actividades instrumentales de la vida diaria (Lawton), instrumento de fluidez verbal (EVP) $e$ instrumento de Reloj. El análisis de datos: descriptiva e inferencial con la utilización de software R. Resultados: después de los talleres de estimulación cognitiva se notó discreto aumento en los puntajes; de los instrumentos de Lawton, Instrumento de Reloj y EVP hubo mantenimiento en los valores; lo que puede ser un indicativo de preservación de la capacidad funcional. Conclusión: se recomienda programas de talleres de estimulación cognitiva con protocolos de intervención sistematizada, con atención específica de las necesidades y seguimiento de la capacidad funcional.

Descriptores: Terapia Cognitiva; Salud del Anciano; Trastornos del Humor; Enfermería Geriátrica; Atención de Enfermería

\section{INTRODUÇÃO}

Com o envelhecimento humano aumentam as queixas de desempenho mnemônico. A deterioração em diferentes áreas da cognição depende de processos fisiológicos que se alteram com a idade, com início e progressão variáveis. As queixas de perda cognitiva podem estar relacionadas aos fatores educacionais, de saúde, bem como, ao nível intelectual global e às capacidades mentais específicas de cada indivíduo. ${ }^{1}$ As desordens mnemônicas são percebidas pelos idosos, especialmente quando comparam seu desempenho atual com o passado. ${ }^{2}$ Além das dificuldades em armazenar informações e resgatá-las, referem ainda prejuízo ocupacional e social. ${ }^{1-2}$

Entretanto, a ausência de queixas de comprometimento de memória, bem como, a manutenção do desempenho cognitivo tem sido considerado um dos indicadores do envelhecimento bem sucedido. ${ }^{3}$ As projeções indicam que em 2050 a população brasileira 


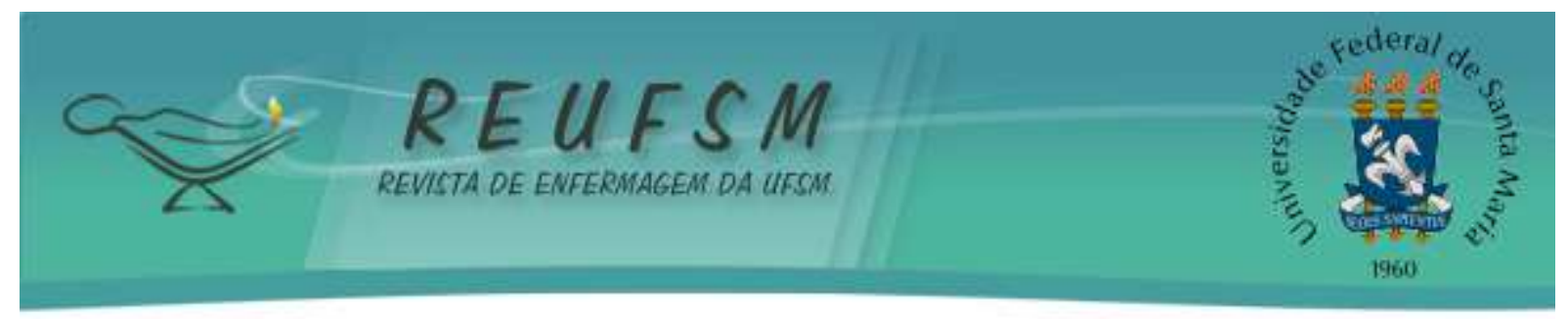

será de 253 milhões de habitantes, a quinta maior população do planeta, abaixo apenas da Índia, China, EUA e Indonésia. E associado ao aumento populacional tem-se as mudanças no panorama de saúde e socioeconômico da população. Esses fatores contribuem significativamente para o aumento da população idosa brasileira. Logo, intervenções que promovam a manutenção da capacidade funcional e a participação social dos idosos devem ser investigadas e desenvolvidas. ${ }^{4-5}$

O conjunto de ideias e sentimentos a respeito da memória denomina-se metamemória. Alterações particulares na metamemória do idoso, como crenças negativas sobre a memória, podem influenciar variáveis moduladoras do desempenho, tais como, esforço despendido, motivação e uso de estratégias mnemônicas. ${ }^{6}$ Nesse quadro se encaixam os idosos com queixa de humor, mas não necessariamente depressão, com resultados na Escala de Depressão Geriátrica (EDG) abaixo de 4, com queixas depressivas associadas a perdas decorrentes do processo de envelhecimento, como isolamento social, sentimento de perda, vigor físico, aparência estética e status social, ou seja, apesar de não serem diagnosticados com depressão, muitas vezes, ressaltam as queixas de perda de memória, quando na verdade possuem queixas de humor. ${ }^{6-7}$

Nesses casos, intervenções conjuntas para queixa de humor e de memória deveriam ser usadas em conjunto, principalmente, para acompanhar sua evolução de tratamento específico para depressão e demência. Essas ações de monitoramento e intervenção são atividades da prática avançada em gerontologia que carecem de estudos sistematizados para construção de evidências científicas, tal como proposto nesse estudo. As intervenções testadas e a metodologia de inclusão dos idosos no grupo podem ser replicadas pelos enfermeiros da prática.

Estima-se que cerca de 11,2 milhões de pessoas receberam diagnóstico de depressão nos últimos anos. Dentre esses, verificou-se maior prevalência sobre pessoas do sexo feminino, totalizando $10,9 \%$, e 3,9\% em homens. Obteve-se ainda que a faixa etária mais susceptível a depressão foi a de idosos de 60 a 64 anos de idade (11,1\%), enquanto o menor percentual foi de jovens de 18 a 29 anos de idade $(5,2 \%) .{ }^{1}$ Dos indivíduos idosos que receberam o diagnóstico, 52\% afirmam usar medicamentos, 16,4\% fazem psicoterapia e 46,4\% receberam assistência médica nos últimos 12 meses. Porém, atendimentos comunitários e primários ainda são restritos, e a enfermagem com prática avançada em gerontologia pode oferecer este tipo de cuidado. ${ }^{1,5}$ 


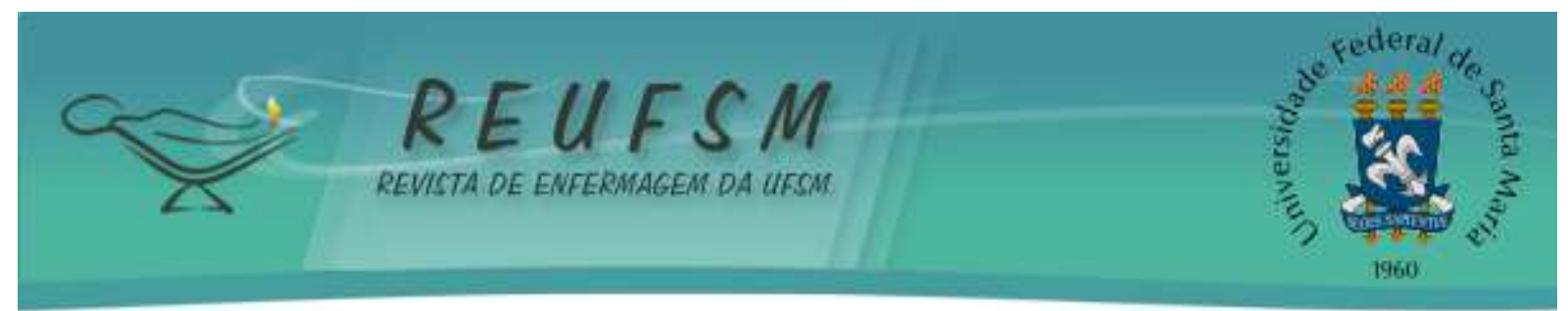

Os grupos de intervenção para idosos são desenvolvidos com a finalidade de promoção do bem-estar subjetivo, visam à promoção de um envelhecimento ativo, potencializar a participação em discussões acerca da saúde, bem-estar físico, social e mental, bem como, permitir a socialização e a criação de vínculos. Dessa forma, auxiliam na preservação da capacidade funcional e do potencial do idoso, a fim de produzir comportamentos saudáveis. ${ }^{5-9}$

As oficinas de estimulação cognitiva ou oficinas terapêuticas, visam estimular o idoso à realização de atividades voltadas para a memória e para o exercício das funções cognitivas. ${ }^{6-}$ 7 Como objetiva a melhora funcional na vida diária, utiliza-se de estratégias: de reminiscências; dinâmicas lúdicas; ou por treino cognitivo, que tem como objetivo a melhora no desempenho de uma tarefa e/ou função cognitiva mediante o ensino de estratégias que o idoso pode utilizar em seu cotidiano. ${ }^{8-9}$

Por meio da percepção de melhora nas atividades de memória, cria-se uma sensação de bem-estar subjetivo, e com isso, abertura com a pessoa idosa para a participação nas intervenções grupais, como dinâmicas de revisão de vida e reminiscências, que foram adicionadas as de estimulação cognitiva, tais quais, intervenções para a melhoria das queixas de humor. Logo, o presente estudo teve como pergunta de pesquisa: ocorre melhora nos testes de avaliação da capacidade funcional do idoso com queixa subjetiva de humor e memória após 14 sessões de intervenção em oficinas de estimulação cognitiva? Para isso, traçou-se como objetivo: analisar um programa de oficinas de estimulação cognitiva para idosos com queixas subjetivas de memória e humor e associá-lo ao desempenho da capacidade funcional.

\section{MÉTODO}

Trata-se de um estudo quase-experimental, com abordagem quantitativa, realizado em um programa para idosos de uma Universidade Pública do Rio de Janeiro, Brasil.

Os procedimentos de coleta de dados ocorreram em três etapas: 1) avaliação préintervenção (testes de rastreio); 2) oficinas de estimulação cognitiva; e 3) avaliação pósintervenção. A avaliação pós-intervenção foi realizada após o encerramento das 14 semanas de atividades desenvolvidas com os idosos. O período foi de julho de 2016 a fevereiro de 2017.

O local de estudo foi nas dependências da Universidade Federal Fluminense, denominado de Espaço Avançado; trata-se de um projeto de extensão multidisciplinar, 


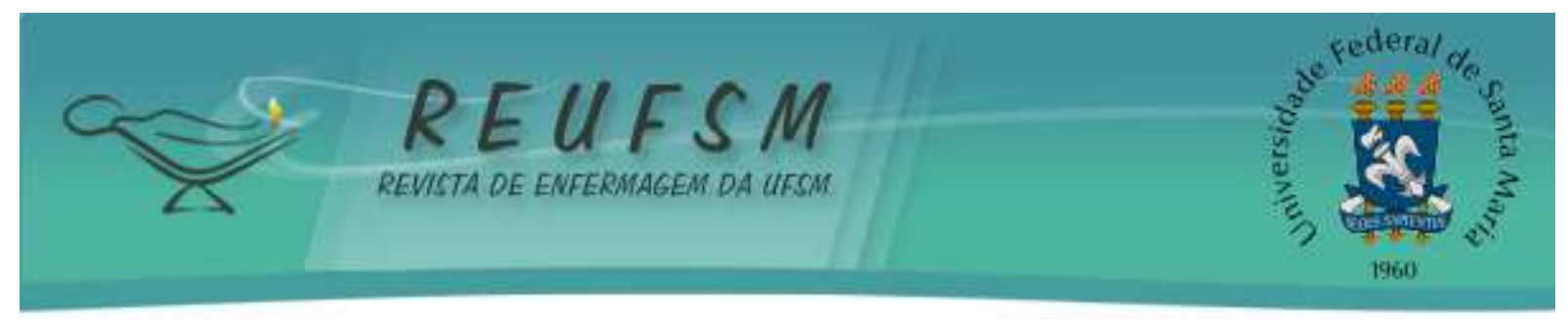

implantado no ano de 1994, na Escola de Serviço Social, que objetiva promover debates sobre questões sociais e trocas de experiências entre idosos ativos e independentes. Em média, são cadastrados anualmente 350 idosos no Programa e, participam diariamente 70 idosos, que têm à sua disposição, cinco dias na semana: hidroginástica; musculação; oficinas de estimulação cognitiva; teatro; yoga; prevenção de quedas; arteterapia; dentre outras atividades.

Foram critérios de inclusão para a pesquisa: ter idade maior ou igual a 60 anos; participar das oficinas do projeto de extensão; apresentar independência para Atividades de Vida Diária; e queixas de humor e depressão (Escala de Depressão Geriátrica $\geq 4$ ); MiniExame do Estado Mental com pontuação acima de 26. E como critérios de exclusão: idosos com igual ou superior de três faltas nas oficinas; diagnóstico médico de depressão em tratamento; luto recente.

Definiu-se como variáveis: idade; sexo; arranjo familiar; grau de escolaridade; e testes amplamente utilizados em gerontologia, Mini Exame de Estado Mental (MEEM), Escala de Depressão Geriátrica (EDG), Escala de Atividades Instrumentais de Vida Diária (Lawton), Teste de Fluência Verbal (EVP) e Teste do Relógio, detalhados a seguir.

O MEEM trata-se de uma avaliação neuropsicológica da função cognitiva. É composto por questões tipicamente agrupadas em 7 categorias, cada uma delas com o objetivo de avaliar funções cognitivas específicas: orientação para tempo (5 pontos); orientação para local (5 pontos); registro de 3 palavras (3 pontos); atenção e cálculo (5 pontos); lembrança das 3 palavras ( 3 pontos); linguagem ( 8 pontos); e capacidade construtiva visual (1 ponto). O escore do MEEM pode variar de um mínimo de 0 até um total máximo de 30 pontos, apresentando boa sensibilidade e especificidade para identificar casos de demência e menos sensível para identificação de casos de Comprometimento Cognitivo Leve. ${ }^{10}$

A Escala de Lawton avalia o desempenho funcional do idoso em relação às suas Atividades Instrumentais de Vida Diária (AIVDS), as quais os possibilitam uma rotina independente. Possui oito atividades que foram avaliados de acordo com o seu desempenho ou participação, e incluem: o uso do telefone, fazer compras, a preparação de refeições, o trabalho doméstico, lavar a roupa, a locomoção fora de casa, a responsabilidade com a medicação, e o controle da economia doméstica. Os itens são classificados quanto à assistência, à qualidade da execução e a iniciativa do participante. Assim, este instrumento fornece informações referentes à relação dependência/independência de uma maneira global e específica nas AIVDS. ${ }^{11}$ 


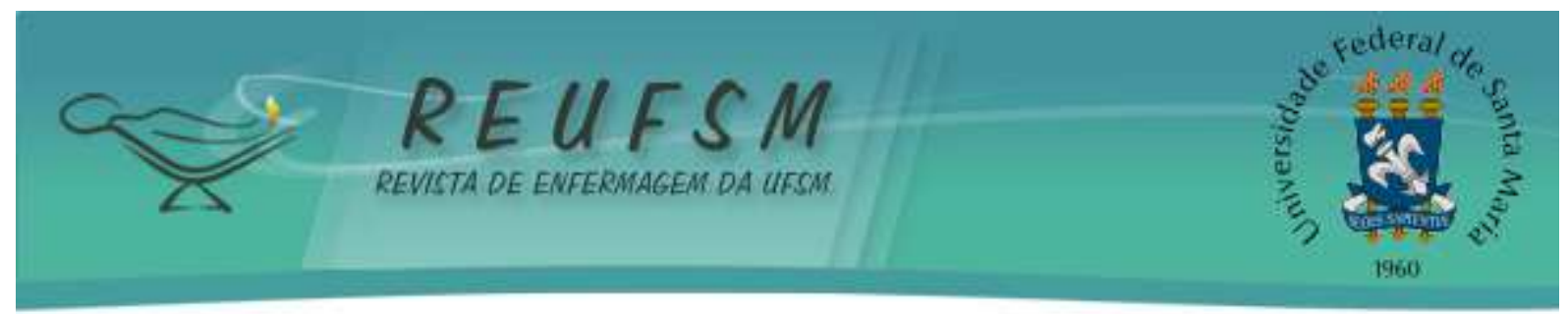

A EDG é um dos instrumentos frequentemente usado na detecção de depressão e queixas de humor na pessoa idosa. É um teste que oferece medidas confiáveis para a avaliação de transtornos depressivos. Em relação ao ponto de corte do EDG, quando o resultado apresentar entre 5 e 7 pontos, deve-se investigar as queixas de humor, quando acima de 7 , constata-se um quadro sugestivo à depressão. ${ }^{12}$

No teste de Fluência Verbal, solicita-se ao participante falar o maior número de animais em 1 minuto. Espera-se uma pontuação entre 13 e 18 animais, sendo desconsideradas as repetições de animais pertencentes à mesma classe. ${ }^{13}$

No teste do relógio é avaliado principalmente as funções executivas e habilidade visual-construtiva. Nesse teste, é analisado se os idosos conseguem desenhar e planejar a circunferência, os números, a posição correta e a hora certa. Apresenta como pontuação máxima 4 pontos, sendo 1 ponto para cada item descrito. ${ }^{14}$

De modo cego, os testes foram realizados por pessoas distintas aos executores das intervenções testadas. Enfermeiros especialistas em Gerontologia, participantes do projeto realizaram os testes. As intervenções foram realizadas por um enfermeiro especialista na área de gerontologia e uma doutora em enfermagem, especialista em psicogeriatria. Haviam ainda o suporte de uma equipe multidisciplinar, caso houvesse necessidade de atendimento dos participantes ao longo da pesquisa para o Serviço Social, a Psicologia ou a Medicina.

Foram dispostos para amostra do estudo 12 idosos selecionados a partir da pontuação alcançada nos testes aplicados; sobretudo, a partir do resultado do EDG, foco central desse estudo. Estes idosos foram alocados em uma oficina específica a fim de se realizar um trabalho direcionado para as necessidades deles.

As oficinas foram realizadas ao longo de 14 semanas, uma vez por semana, com duração de 60 minutos, e foram planejadas previamente, atendendo as necessidades do perfil do grupo. A Tabela 1 apresenta a temática das oficinas, bem como, a área cognitiva treinada. 


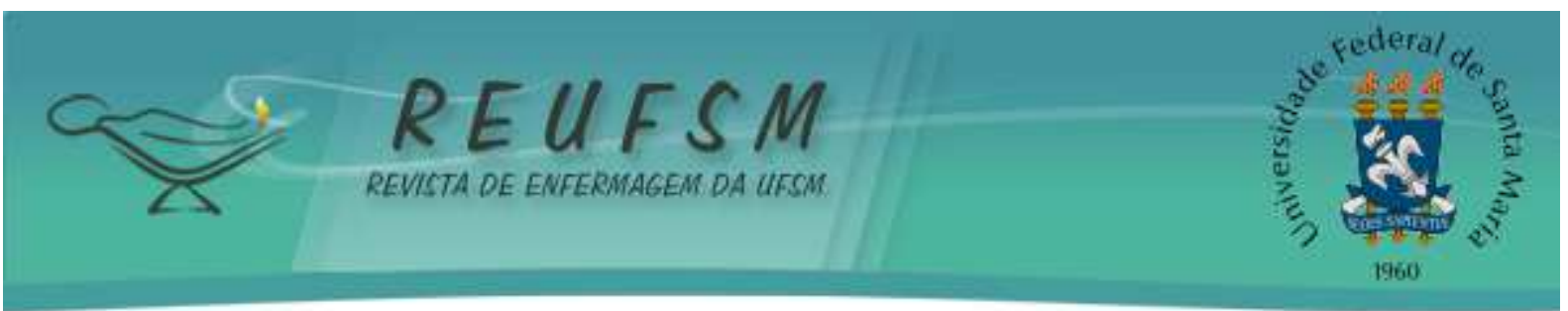

Tabela 1 - Intervenção quanto à área cognitiva treinada e tipo de atividade. Niterói/RJ, Brasil, 2017

\begin{tabular}{|c|c|c|c|c|}
\hline Semana & Atividade & Individual & Coletiva & Área cognitiva treinada \\
\hline 1 & $\begin{array}{l}\text { Sopa de letras: alfabeto } \\
\text { completo em PVC }\end{array}$ & $\checkmark$ & $\checkmark$ & $\begin{array}{l}\text { Memória de longo prazo; associação de ideias; } \\
\text { diálogo; cooperação entre os sujeitos; interação } \\
\text { entre sujeitos. }\end{array}$ \\
\hline 2 & Colocando na certa & $\checkmark$ & $\checkmark$ & $\begin{array}{l}\text { Noção de sequência; associação de ideias; } \\
\text { atenção/concentração; estímulo de trabalho em } \\
\text { grupo; categorização; memória recente. }\end{array}$ \\
\hline 3 & Expondo as emoções & $\checkmark$ & $\checkmark$ & $\begin{array}{l}\text { Criatividade, associação de ideias, atenção, } \\
\text { concentração, memória de longo prazo e } \\
\text { memória recente. }\end{array}$ \\
\hline 4 & Jornal da vida & $\checkmark$ & $\checkmark$ & $\begin{array}{l}\text { Interação grupal, construção em grupo, } \\
\text { expressão, memória recente, memória de longo } \\
\text { prazo. }\end{array}$ \\
\hline 5 & Abrindo o jogo & $\checkmark$ & $\checkmark$ & $\begin{array}{l}\text { Atenção, concentração, lateralidade, raciocínio } \\
\text { lógico, leitura, expressão oral de ideias. }\end{array}$ \\
\hline 6 & Organizando as palavras & $\checkmark$ & $\checkmark$ & $\begin{array}{l}\text { Atenção, concentração, raciocínio lógico, leitura, } \\
\text { interação grupal ao dividir sua opinião sobre a } \\
\text { frase. }\end{array}$ \\
\hline 7 & Música em roda & $\checkmark$ & $\checkmark$ & $\begin{array}{l}\text { Interação grupal, memória de curto prazo, } \\
\text { memória de longo prazo, atenção, concentração. }\end{array}$ \\
\hline 8 & Jogo dos erros & $\checkmark$ & $\checkmark$ & $\begin{array}{l}\text { Percepção visual, atenção, concentração, } \\
\text { criatividade, memória recente, associação de } \\
\text { ideias, expressão oral. }\end{array}$ \\
\hline 9 & Labirinto & $\checkmark$ & $\checkmark$ & Atenção, concentração, raciocínio lógico. \\
\hline 10 & O que há de diferente? & $\checkmark$ & $\checkmark$ & $\begin{array}{l}\text { Percepção visual de semelhanças e diferenças, } \\
\text { noção de figura e fundo, atenção, concentração. }\end{array}$ \\
\hline 11 & Ditos populares & $\checkmark$ & $\checkmark$ & $\begin{array}{l}\text { Atenção, concentração, memória associativa, } \\
\text { compreensão de metáforas, raciocínio analógico, } \\
\text { expressão oral, leitura. }\end{array}$ \\
\hline 12 & Do que você lembra? & $\checkmark$ & $\checkmark$ & $\begin{array}{l}\text { Estímulo da atenção e da concentração, estímulo } \\
\text { visual e da figura-fundo, estímulo da memória } \\
\text { imediata e recente. }\end{array}$ \\
\hline 13 & Caça palavras & $\checkmark$ & $\checkmark$ & Atenção, concentração, memória de curto prazo. \\
\hline 14 & $\begin{array}{l}\text { O que há de errado na } \\
\text { ilustração? }\end{array}$ & $\checkmark$ & $\checkmark$ & Atenção, concentração. \\
\hline
\end{tabular}

As oficinas de estimulação cognitiva foram fundamentadas na taxonomia de Classificação das Intervenções de Enfermagem (NIC), Domínio 3 - Comportamental, e a 


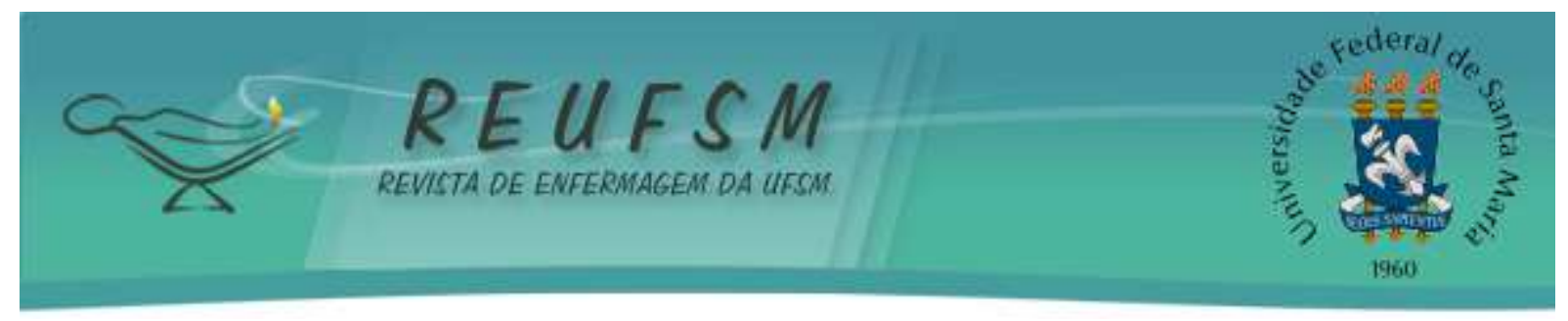

classe P - Terapia Cognitiva, que tem por definição Intervenções para reforçar ou promover comportamentos desejáveis, ou alterar comportamentos indesejáveis. ${ }^{15}$

Ainda, após a finalização das 14 sessões foi utilizado um instrumento de autoavaliação dos participantes quanto ao seu desempenho e a estrutura das oficinas de estimulação cognitiva, preenchido individualmente, contendo oito questões abertas e fechadas, dispostas em uma única lauda de papel com letras apropriadas. Somente um idoso solicitou ajuda para o preenchimento por dificuldade visual.

Para análise dos dados, organizou-se um banco de dados no programa Excel, e a utilização do software R. Nas comparações de diferença no tratamento pré e pós intervenção foi utilizado o teste não-paramétrico de Wilcoxon, que tem como finalidade testar a igualdade das medidas de posição de duas amostras dependentes. O nível de significância $\alpha$ foi fixado como $\alpha=0.05$.

A presente pesquisa foi realizada em conformidade com os princípios éticos para pesquisa com seres humanos, apresentados na resolução 446/12 do Conselho Nacional de Saúde e obteve aprovação junto ao Comitê de Ética em Pesquisa, sob parecer de número 250.132 em 05/04/2013. Todos os participantes da pesquisa foram informados quanto aos objetivos, e, após foi assinado o Termo de Consentimento Livre e Esclarecido.

\section{RESULTADOS}

Quanto a caracterização amostral, obteve-se um total de 12 participantes, sendo 11 do sexo feminino $(91,6 \%)$ e um do sexo masculino $(8,4 \%)$; referente a idade, cinco $(41,6 \%)$ apresentaram idade entre 60 e 69 anos, e sete $(58,3 \%)$ indivíduos com idade entre 70 e 89 anos. Ao que se refere ao arranjo familiar, nove $(75,0 \%)$ apresentaram unipessoalidade domiciliar e três $(25,0 \%)$ vivem com duas pessoas ou mais. Em relação à escolaridade, cinco $(41,6 \%)$ possuíam o ensino médio completo, e igualmente três $(25,0 \%)$ ensino fundamental completo, e ensino superior completo. A Tabela 2 detalha as variáveis Pré e Pós intervenção. 


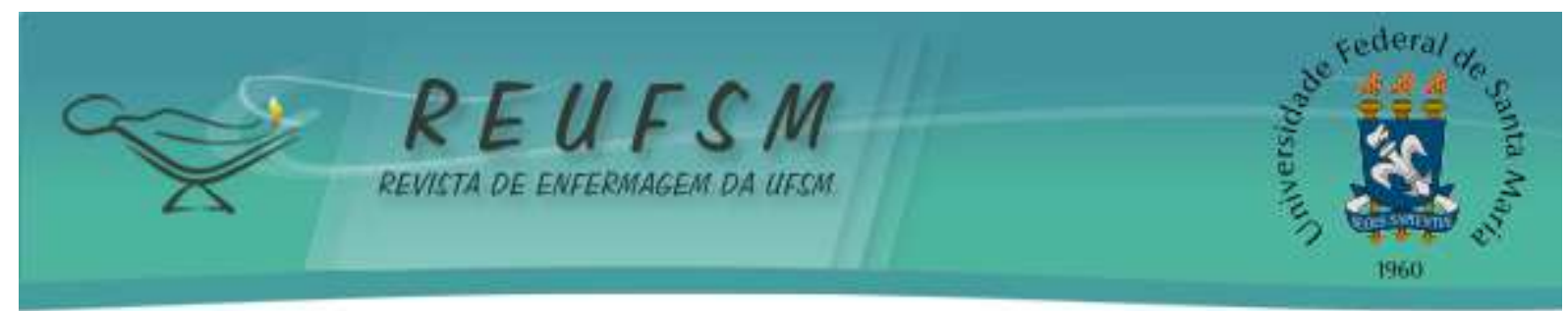

Tabela 2 - Distribuição dos escores das variáveis testadas Pré e Pós intervenção. Niterói/RJ, Brasil, 2017

\begin{tabular}{|c|c|c|c|c|c|c|}
\hline & Variável & Média & & $\begin{array}{l}\text { Desvio } \\
\text { padrão }\end{array}$ & Mediana & p-valor \\
\hline \multirow{4}{*}{ MEEM } & Pré & 26,58 & \pm & 2,43 & 27 & \\
\hline & Pós & 27,25 & \pm & 2,01 & 27 & \\
\hline & Variação Absoluta & $-0,67$ & \pm & 1,72 & -1 & 0,18 \\
\hline & Variação Relativa (\%) & 0,00 & \pm & 0,07 & $-0,01$ & 0,78 \\
\hline \multirow{4}{*}{ EDG } & Pré & 4,75 & \pm & 3,14 & 5 & \\
\hline & Pós & 4,00 & \pm & 2,56 & 3 & \\
\hline & Variação Absoluta & 0,75 & \pm & 3,08 & 0,5 & 0,48 \\
\hline & Variação Relativa (\%) & 0,00 & \pm & 0,69 & $-0,07$ & 0,91 \\
\hline \multirow{4}{*}{ LAWTON } & Pré & 20,83 & \pm & 0,39 & 21 & \\
\hline & Pós & 20,67 & \pm & 0,65 & 21 & \\
\hline & Variação Absoluta & 0,17 & \pm & 0,58 & 0 & 0,42 \\
\hline & Variação Relativa (\%) & 0,00 & \pm & 0,03 & $-0,01$ & 0,49 \\
\hline \multirow{4}{*}{ RELÓGIO } & Pré & 2,75 & \pm & 1,06 & 3 & \\
\hline & Pós & 2,83 & \pm & 1,27 & 3 & \\
\hline & Variação Absoluta & $-0,08$ & \pm & 1,51 & 0 & 0,89 \\
\hline & Variação Relativa (\%) & 0,00 & \pm & 0,54 & 0,03 & 0,91 \\
\hline \multirow{4}{*}{ EVP } & Pré & 15,67 & \pm & 6,01 & 14 & \\
\hline & Pós & 15,92 & \pm & 4,38 & 17 & \\
\hline & Variação Absoluta & $-0,25$ & \pm & 5,66 & 0 & 0,88 \\
\hline & Variação Relativa (\%) & 0,00 & \pm & 0,36 & 0,02 & 1,00 \\
\hline
\end{tabular}

Nota-se um sutil aumento do escore do MEEM (pré 26,58 $\pm 2,43$ e pós 27,25 $\pm 2,01$ ), e uma diminuição no escore do EDG (pré 4,75 $\pm 3,14$ e pós 4,00 $\pm 2,56$ ). Já nos escores dos testes de Lawton, Teste do Relógio e EVP observa-se uma manutenção nos valores, o que pode ser um indicativo de preservação nas atividades de vida diária. Dados esses, de relevância clínica, pois apesar dos p-valores demonstrarem que não houve diferença significativa, apontam também, que não houve piora, o que indica a manutenção da capacidade funcional.

Com relação a avaliação das atividades, os idosos realizaram uma autoavaliação, que foi aplicada como meio de avaliar subjetivamente, ao final de cada semestre, se as oficinas correspondiam ao objetivo proposto, como também para aperfeiçoar o serviço prestado, conforme apontado na Tabela 3. 


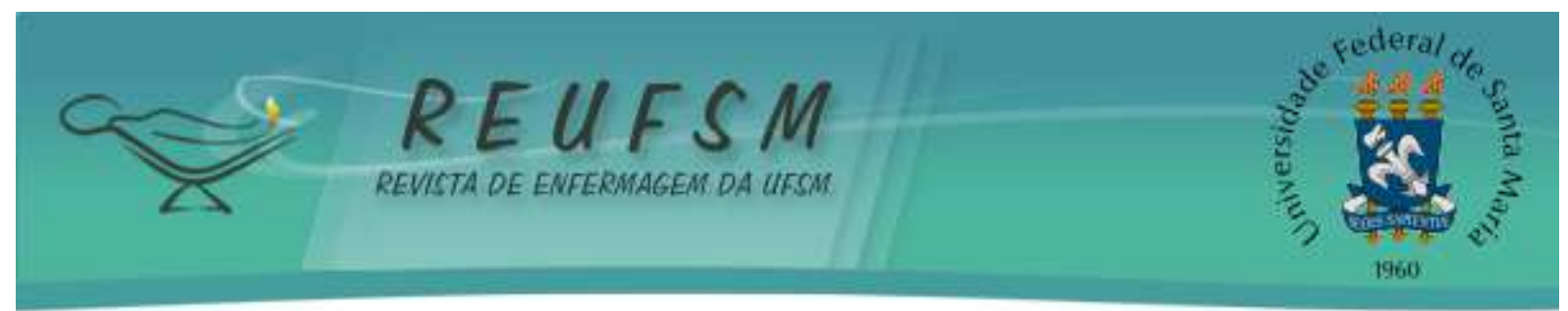

Tabela 3 - Autoavaliação dos participantes quanto ao seu desempenho e estrutura das oficinas. Niterói/RJ, Brasil, 2017

\begin{tabular}{|c|c|}
\hline Autoavaliação & n $(\%)$ \\
\hline \multicolumn{2}{|c|}{ Sentiu-se seguro quanto à compreensão do conteúdo? } \\
\hline Sim & $10(83,3)$ \\
\hline \multicolumn{2}{|c|}{ Foi participativo durante as atividades? } \\
\hline Sim & $12(100,0)$ \\
\hline \multicolumn{2}{|c|}{ Interagiu com os colegas? } \\
\hline Sim & $12(100,0)$ \\
\hline \multicolumn{2}{|c|}{$\begin{array}{l}\text { Em uma escala de zero a } 5 \text {, sendo que zero corresponde a nenhuma melhora e } \\
5 \text { corresponde a uma melhora total, qual opção corresponde à melhora sentida } \\
\text { quanto as queixas de memória? }\end{array}$} \\
\hline 2 & $1(8,3)$ \\
\hline 3 & $1(8,3)$ \\
\hline 4 & $2(16,6)$ \\
\hline 5 & $8(66,6)$ \\
\hline \multicolumn{2}{|c|}{ Avaliação das oficinas } \\
\hline \multicolumn{2}{|c|}{ O tempo de duração das oficinas é satisfatório? } \\
\hline Sim & $9(75,0)$ \\
\hline Não & $3(25,0)$ \\
\hline \multicolumn{2}{|c|}{ Qual seu grau de satisfação com relação às oficinas? } \\
\hline Ótimo & $11(91,6)$ \\
\hline Bom & $1(08,3)$ \\
\hline \multicolumn{2}{|c|}{$\begin{array}{l}\text { Os materiais usados durante a realização das oficinas foram satisfatórios/eram } \\
\text { de fácil entendimento? }\end{array}$} \\
\hline Em todas & $9(75,0)$ \\
\hline Parcialmente & $3(25,0)$ \\
\hline \multicolumn{2}{|c|}{ A proposta das oficinas cumpriu seus objetivos? } \\
\hline Sim & $12(100,0)$ \\
\hline
\end{tabular}

Observa-se que $100 \%$ dos idosos $(n=12)$ alegam ter interagido com os colegas do grupo e se consideraram participativos durante as atividades. Estes dados denotam a interação e a cooperação entre os sujeitos algo a se considerar na melhoria de um quadro de humor. Como também expressão a melhoria na memória, observado em $66 \%$ dos idosos, em relação à avaliação das oficinas, $75 \%$ estão satisfeitos com o material utilizado e $100 \%$ afirma que as oficinas cumpriram com os objetivos. Esse feedback permite dar continuidade a intervenção específica proposta, que visa dois objetivos terapêuticos, o bem-estar psicológico dos idosos e a manutenção dos processos cognitivos. 


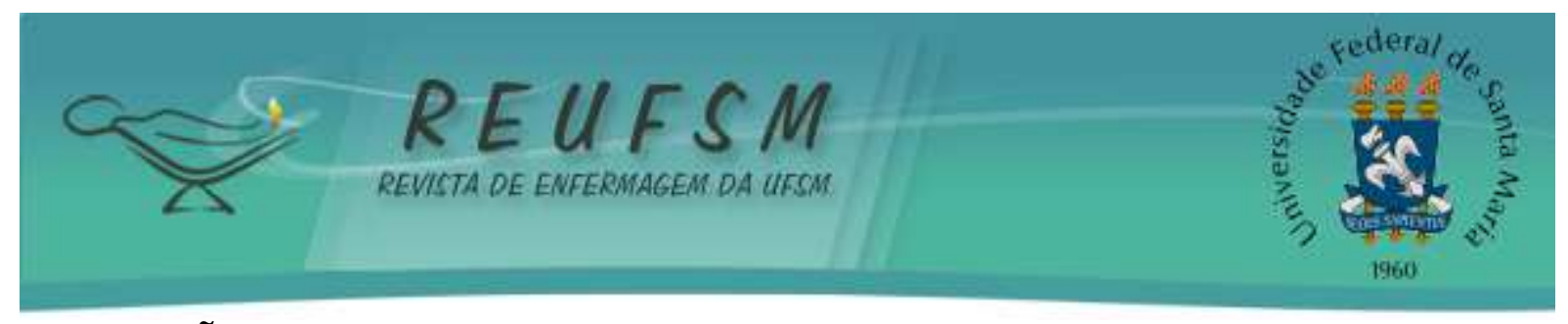

\section{DISCUSSÃO}

O principal dado desse estudo refere-se a especificidade do grupo estudado, ou seja, associação de queixas subjetivas de humor e memória. Um grupo como esse requer uma abordagem integrada de ações para ativação da memória e sobre os aspectos psicossociais como humor, perdas, autoestima e isolamento. ${ }^{16}$ A correlação entre os sintomas depressivos e as queixas de memória foi destaque numa pesquisa com uma amostra de 204 idosos, que encontrou relação estatisticamente significante entre a queixa subjetiva de comprometimento de memória e fatores emocionais, como sintomas de depressão e autoestima. ${ }^{17}$

Os resultados demonstraram que não houve diferença significativa na variável EDG. Ainda assim, pode-se observar uma tendência à queda da pontuação. Isto pode indicar que as oficinas de estimulação cognitiva colaboraram no tratamento das queixas de humor e depressão desses idosos. Por isso, ações que valorizam as queixas subjetivas dos idosos, com avaliação sistematizada e planejamento das ações de enfermagem direcionadas ao alcance de objetivos devem ser almejadas, em detrimento de ações grupais inespecíficas, sem seguimento dos escores de capacidade funcional, em planejamento de intervenções individualizadas, que supostamente rotulam os idosos como depressivos ou com perda de memória.

No que tange às características sociodemográficas dos participantes, observou-se a predominância do sexo feminino, resultado coerente com estudo, segundo o qual, a população do sexo feminino era de $90,8 \% .{ }^{18}$ Ressalta-se como fundamental que as atividades propostas no grupo sejam direcionadas para ambos os sexos, apesar do baixo número amostral de indivíduos do sexo masculino, isso evita a evasão dos mesmos.

Outro fator importante para analisar no perfil dos indivíduos é a faixa etária, observouse predominância dos idosos de 70 a 79 anos. A despeito da idade avançada, o diagnóstico de depressão e as queixas de humor podem ser agravantes para o mau funcionamento cognitivo, comprometer a qualidade de vida e o desenvolvimento das atividades de vida diária desses indivíduos. $^{18-19}$

A idade também deve ser avaliada quanto aos resultados obtidos no teste de rastreio MEEM. Idosos com idade entre 71 e 74 anos obtiveram pontuação mais alta, quando comparados aos indivíduos com 80 anos ou mais. ${ }^{19}$ Esse fato pode ratificar um resultado expressivo da estabilização dos dados da variável MEEM para as diferentes faixas etárias, devido a prevalência de indivíduos entre 70 e 89 anos. 


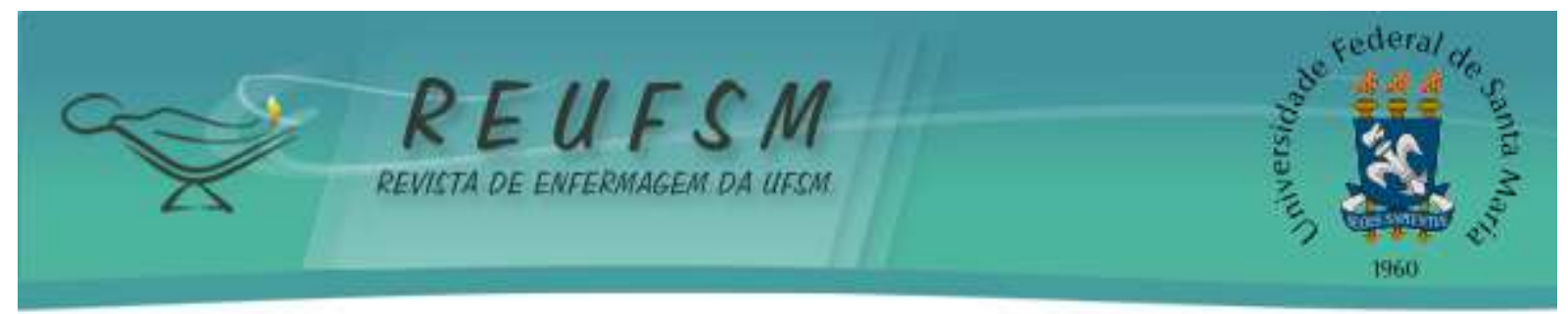

Outro resultado que merece destaque é a unipessoalidade doméstica, ou seja, idosos que moram sozinhos. $\mathrm{O}$ isolamento social, as perdas recorrentes de entes familiares e da rede de apoio social são importantes itens de avaliação nas consultas de enfermagem. Como também necessitam fazer parte do plano de ação da enfermeira e equipe multidisciplinar a promoção e a prevenção de suicídio e institucionalizações precoces. ${ }^{4,7}$

O comportamento de estabilização dos dados das escalas de Lawton, EVP e Teste do Relógio podem expressar a importância da avaliação sistemática, além daa realização da intervenção para essa população, uma vez que a idade avançada tende a causar prejuízos cognitivos. ${ }^{18-19}$ Ademais, preservar a cognição e a capacidade de realizar as atividades de vida diária podem contribuir para a melhora dos sintomas da depressão, ao promover no idoso autonomia e independência.

Para o desenvolvimento das intervenções fez-se necessário construir atividades direcionadas, de modo a estimular não somente os processos cognitivos, mas também a interação com os outros idosos. O trabalho em equipe, a confiança, autoestima, comunicação, e criatividade proporcionam relaxamento, melhora do humor, motivação e diminuição da ansiedade. ${ }^{20} \mathrm{O}$ trabalho interdisciplinar com a psicologia, que dava suporte se houvesse casos de crises ou necessidade de encaminhamentos, também se mostrou relevante para aplicação dessas intervenções, apesar de não ter sido necessário.

A autoavaliação dos idosos deve ser analisada, no que se refere à percepção dos mesmos, perante a interação com os outros integrantes do grupo. Este fato corrobora com uma pesquisa, a qual afirma que o fato dos idosos interagirem com outros de sua faixa etária os motiva a participar de grupos de convivência. ${ }^{21}$

A implementação de cuidados pelos profissionais de enfermagem aos idosos, objetivando melhorar sua qualidade de vida, vem auxiliando na qualidade do envelhecimento. Isto tem incentivado a realização de estudos que caracterizem melhor as deficiências dos idosos e investiguem as intervenções de enfermagem que mais se aplicam às situações clínicas específicas desta população. ${ }^{22}$

\section{CONCLUSÃO}

A análise da performance em testes funcionais e avaliação subjetiva da intervenção proposta de estimulação cognitiva para pessoas idosas com queixas subjetivas de humor e 


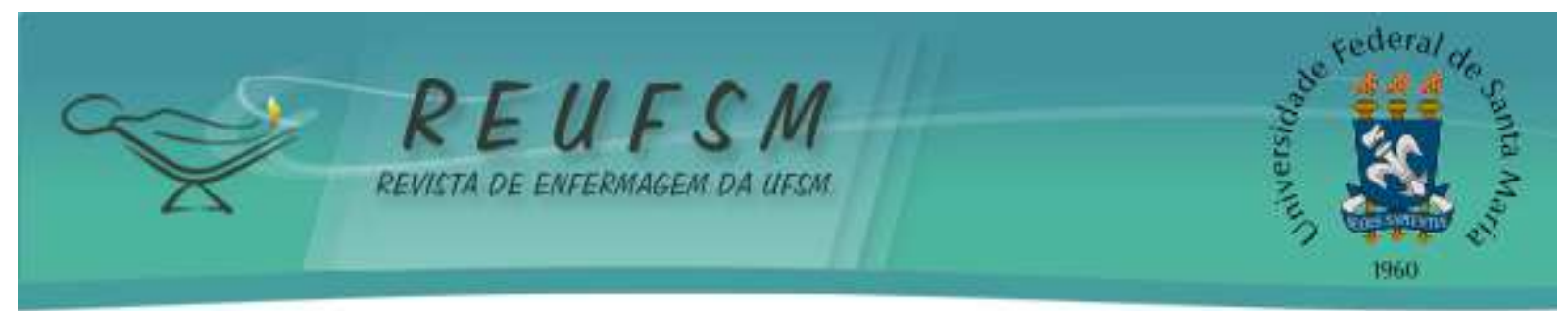

memória, demonstrou manutenção da capacidade funcional e uma tendência de queda na pontuação da Escala de Depressão Geriátrica, resultado esse considerado positivo, dado o pequeno período de tempo de seguimento e de intervenção. Pela autoavaliação, observou-se o benefício no reestabelecimento do convívio social, o que pode também ter influenciado os resultados dos testes.

Das limitações encontradas podem-se citar: o tamanho reduzido da amostra, porém o tipo de intervenção testada em grupo limita o número ampliado de participantes no mesmo grupo; a ausência de um grupo controle; distinção da medicação utilizada para distúrbios depressivos; e a utilização do EDG como único teste de rastreio para queixas de humor. É fundamental que se utilize outros instrumentos de medida validados para índices de ansiedade e estresse, desse modo, seria possível avaliar os idosos com maior precisão e validade. Como também, recomenda-se novos estudos com maior tempo de seguimento e dias de intervenção.

No entanto, recomenda-se investir em programas de intervenção sistematizadas para idosos com queixa subjetiva de memória e humor, que garantam atendimento específico às suas demandas, e seguimento de sua performance funcional. Faz-se necessário diferenciar a heterogeneidade de necessidades da população idosa, pessoas com queixas de humor, de memória, ou de ambos, que necessitam de grupos direcionados e pré-selecionados, com intervenções assertivas e monitorização da capacidade funcional e subjetiva ao longo do programa de intervenção.

\section{REFERÊNCIAS}

1. Silva LSV, Silva TBL, Falcão DVS, Batistoni SST, Lopes A, Cachioni M, et al. Relações entre queixas de memória, sintomas depressivos e desempenho cognitivo em idosos residentes na comunidade. Rev Psiq Clín [Internet]. 2014 [acesso em 2017 jun 13];41(3):67-71. Disponível em: http://taurus.unicamp.br/bitstream/REPOSIP/87647/1/2-s2.084906082839.pdf.

2. Holz AW, Nunes BP, Thumé E, Lange C, Facchini LA. Prevalência de déficit cognitivo e fatores associados entre idosos de Bagé, Rio Grande do Sul, Brasil. Rev Bras Epidemiol. [Internet]. 2013 [acesso em 2017 jun 13];16(4):880-8. Disponível em: http://www.scielo.br/pdf/rbepid/v16n4/pt_1415-790X-rbepid-16-04-00880.pdf.

3. Ploner KS, Gomes MC, dos Santos ST. Metamemória no envelhecimento e os impactos promovidos pela Oficina de Memória. Rev Bras Cienc Envel Hum [Internet]. 2016 [acesso em 2017 jun 13];13(2):197-218. Disponível em: http://seer.upf.br/index.php/rbceh/article/view/5937/pdf. 


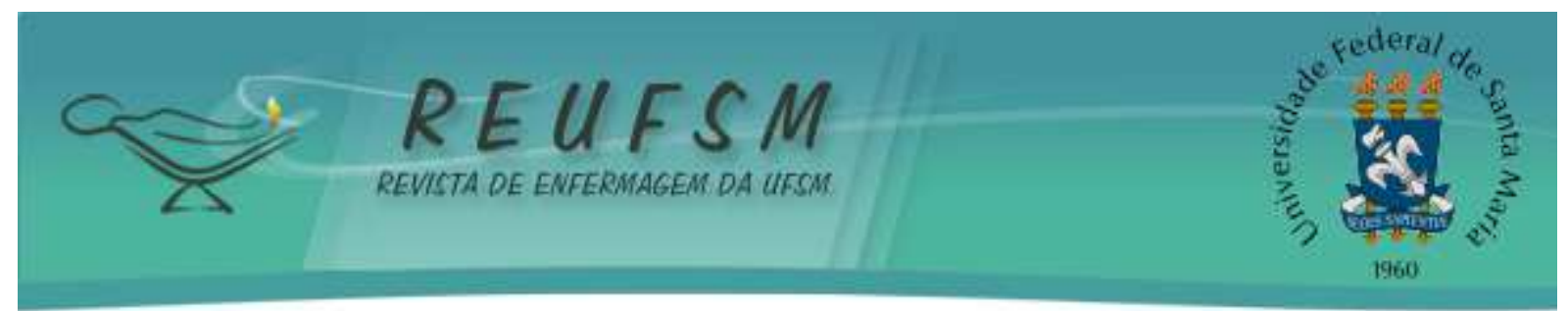

4. Guedes MBOG, Lima KC, Caldas CP, Veras RP. Apoio social e o cuidado integral à saúde do idoso. Physis: Rev Saud Colet [Internet]. 2017 [acesso em 2018 fev 17];27(4):1185-204. Disponível em: https://doi.org/10.1590/S0103-73312017000400017.

5. Brasil GLP, Oliveira EAR, Formiga LMF, Oliveira AKS, Silva RN, Lopes CM. Profile of older persons participating groups of health promotion. Rev Enferm UFPI [Internet]. 2014 [acesso em 2017 jun 13];2(4):28-34. Disponível em: http://www.ojs.ufpi.br/index.php/reufpi/article/view/1197/pdf_1.

6. Finn M, McDonald S. A single case study of computerized cognitive training for older persons with mild cognitive impairment. Neuro Rehabilitation [Internet]. 2014 [acesso em 2017 jun 13];35(2):261-70. Disponível em: https://www.ncbi.nlm.nih.gov/pubmed/24990031.

7. Bahar-Fuchs A, Clare L, Woods B. Cognitive training and cognitive rehabilitation for mild to moderate Alzheimer's disease and vascular dementia. Cochrane Database Syst Rev [Internet]. 2013 jun [accesso em 2017 jun 13];5:(6):CD003260. Disponivel em: https://www.ncbi.nlm.nih.gov/pubmed/23740535.

8. Mowszowski L, Batchelor J, Naismith SL. Early intervention for cognitive decline: can cognitive training be used as a selective preventive technique? Int Psychogeriatr. 2010;22(4):537-48.

9. Santana RF, Rosa TB, Aquino RG, Alexandrino AS, Santos GLA, Lobato HA. Maintenance of functional capacity in cognitive stimulation subgroups. Invest Educ Enferm [Internet]. 2016 [acesso em 2017 jun 13];34(3):493-501. Disponivel em: http://www.scielo.org.co/pdf/iee/v34n3/2216-0280-iee-34-03-00492.pdf.

10. Folstein, M. F., Folstein, S. E., \& McHugh, P. R. (1975). "Mini-mental state". A practical method for grading the cognitive state of patients for the clinician. J Psychiatric Res. 1975; 12(3):189-98.

11. Lawton MP, Brody EM. Assessment of older people: self-maintaining and instrumental activities of daily living. The Gerontologist. 1969;9(3):179-86.

12. Almeida OP, Almeida SA. Confiabilidade da versão brasileira da Escala de Depressão em Geriatria (GDS) versão reduzida. Arq Neuro-Psiquiatr [Internet]. 1999 jun [acesso 2018 mar 13];57(2B):421-6.

Disponível

em:

http://www.scielo.br/scielo.php?script=sci_arttext\&pid=S0004-

282X1999000300013\&lng=en.

13. Santana APO, dos Santos KP. Teste de fluência verbal: uma revisão histórico-crítica do conceito de fluência. Distúrb Comun [Internet]. 2015 [acesso em 2017 jun 13];27(4):807-18. Disponível em: https://revistas.pucsp.br/index.php/dic/article/viewFile/23334/18813.

14. Silva KCA, Lourenço RA. Tradução, adaptação e validação de construto do Teste do Relógio aplicado entre idosos no Brasil. Rev Saúde Pública [Internet]. 2008 [acesso em 2017 jun 13];42(5):930-7. Disponível em: http://www.scielo.br/pdf/rsp/v42n5/5765.pdf.

15. Bulechek GM, Butcher HK, Dochterman JM, Wagner CM. Classificação das Intervenções de Enfermagem (NIC). $6^{\mathrm{a}}$ ed. Rio de Janeiro: Elsevier; 2016. 640 p.

16. Santos AT, Leyendecker DD, Costa ALS, de Souza-Talarico JN. Queixa subjetiva de comprometimento da memória em idosos saudáveis: influência de sintomas depressivos, 


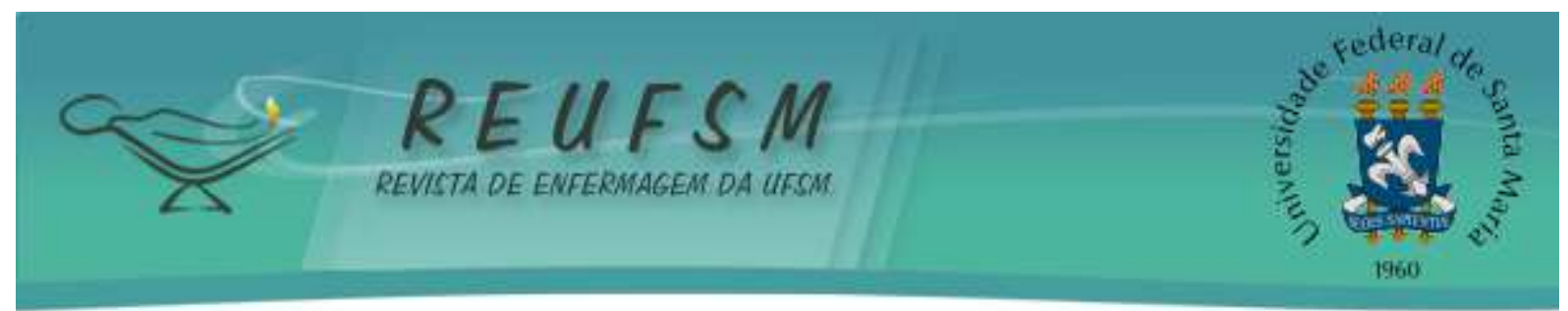

percepção de estresse e autoestima. Rev Esc Enferm USP [Internet]. 2012 [acesso em 2017 jun 13];46(N Esp):24-9. Disponível http://www.scielo.br/scielo.php?script=sci_arttext\&pid=S0080$62342012000700004 \& \operatorname{lng}=$ en $\& n r m=$ iso.

17. Andrade NA, Nascimento MMP, Oliveira MMD, Queiroga RM, Fonseca FLA, Lacerda SNB, et al. Percepção de idosos sobre grupo de convivência: estudo na cidade de CajazeirasPB. Rev Bras Geriatr Gerontol [Internet]. 2014 [acesso em 2017 jun 13];17(1):39-48. Disponível em: <http://www.scielo.br/pdf/rbgg/v17n1/1809-9823-rbgg-17-01-00039.pdf.

18. Almeida AV, Mafra SCT, Silva EP, Kanso S. A feminização da velhice: em foco as características socioeconômicas, pessoais e familiares das idosas e o risco social. Textos Contextos [Internet]. 2015 [acesso em 2017 jun 13];14[1]:115-31. Disponível em: http://revistaseletronicas.pucrs.br/ojs/index.php/fass/article/viewFile/19830/13313.

19. Campos APR, Silva CM, Castro SS, Graminha CV. Depressão e qualidade de vida em indivíduos com artrite reumatoide e indivíduos com saúde estável: um estudo comparativo. Fisioter Pesqu [Internet]. 2013 [acesso em 2017 jun 13];20(4):401-7. Disponível em: http://www.scielo.br/pdf/fp/v20n4/v20n4a16.pdf.

20. Pinheiro PA, Passos TDRO, Coqueiro RS, Fernandes MH, Barbosa AR. Desempenho motor de idosos do Nordeste brasileiro: diferenças entre idade e sexo. Rev Esc Enferm USP [Internet]. 2013 fev [acesso em 2017 jun 13];47(1):128-36. Disponível em: http://www.scielo.br/scielo.php?script=sci_arttext\&pid=S0080$62342013000100016 \& \operatorname{lng}=$ en $\& n r m=$ iso.

21. Nóbrega IRAP, Leal MCC, Marques APO, Vieira JCM. Fatores associados à depressão em idosos institucionalizados: revisão integrativa. Saúde Debate [Internet]. 2015 [acesso em 2017 jun 13];39(105):536-50. Disponível em: http://www.scielo.br/scielo.php?script=sci_arttext\&pid=S0103$11042015000200536 \& \operatorname{lng}=$ en $\& n r m=$ iso.

22. Santana RF, Lobato HA, Santos GLA, Alexandrinho SA, Alencar TD, Souza TA. Oficinas de estimulação cognitiva para idosos com baixa escolaridade: estudo intervenção. Av Enferm [Internet]. 2016 [acesso em 2017 jun 13];34(2):148-58. Disponível em: https://revistas.unal.edu.co/index.php/avenferm/article/view/44711/58008.

Data de submissão: 18/02/2018

Data de aceite: 03/08/2018

Autor correspondente: Rosimere Ferreira Santana

E-mail: rosifesa@gmail.com

Endereço: Rua Dr. Celestino 74 Centro, Niterói, Rio de Janeiro, Brasil.

CEP: 24020-091 